\title{
Recidiva tardia de melanoma cutâneo: relato de caso
}

Diogo Barata de Almeida, ${ }^{1}$ Márcia Gonçalves Lopes ${ }^{2}$

\section{RESUMO}

Introdução: Em doentes com melanoma maligno cutâneo (MMC), a sobrevida após os 10 anos tem sido considerada sinónimo de cura. Contudo, a recidiva após este período, designada como recidiva tardia, tem vindo a ser descrita por alguns autores. Com este caso pretende-se sensibilizar para a importância de estar atento às recidivas tardias do MMC e refletir sobre o papel do médico de família (MF) num diagnóstico atempado das mesmas.

Descrição do caso: Homem de 50 anos, fumador, com história de hipertensão arterial e excisão de MMC da perna aos 28 anos, recorre à MF por tosse seca nos últimos dois dias, sem outra sintomatologia acompanhante. Admitiu-se infeção aguda do aparelho respiratório superior, tendo sido medicado sintomaticamente. Regressa um mês depois, por persistência da tosse, sem outros sinais ou sintomas adicionais. Colocou-se a hipótese de efeito secundário ao Zofenopril que tomava para a hipertensão arterial, tendo sido realizada substituição por Irbesartan. O utente regressa após dois meses, mantendo a queixa inicial. À observação mantinha-se sem alterações relevantes, voltando a ser excluídos sinais de alarme. Trazia, contudo, análises requisitadas pela medicina ocupacional que evidenciavam uma velocidade de sedimentação de $87 \mathrm{~mm} / \mathrm{h}$ sem outras alterações. Admitiu-se eventual tuberculose pulmonar ou neoplasia pulmonar, tendo sido referenciado ao serviço de urgência, onde realizou tomografia computorizada torácica que revelou lesão nodular de características proliferativas no ápex pulmonar esquerdo, compatível com possível processo neoplásico pulmonar. Foi referenciado à consulta de pneumologia e submetido a resseção parcial da massa. Os resultados histológicos mostraram metástase de MMC, 22 anos após o seu diagnóstico inicial.

Comentário: Verifica-se na literatura um número crescente de casos reportando recidivas tardias de MMC, pelo que a sua deteção precoce deverá ser uma preocupação dos clínicos. Considerando a continuidade de cuidados prestada pelo MF, torna-se premente que este desempenhe um papel crucial na monitorização de tais casos.

Palavras-chave: Melanoma; Recidiva tardia.

\section{INTRODUÇÃO}

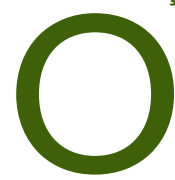

melanoma maligno cutâneo (MMC) é uma neoplasia que tem origem nos melanócitos. ${ }^{1}$ É mais frequente entre os 30 e 60 anos de idade, ${ }^{1}$ apresentando maior incidência nos indivíduos de pele clara que vivem a baixas latitudes. ${ }^{2}$

A história de exposição a radiação ultravioleta (em particular na infância), a presença de antecedentes familiares ou pessoais de MMC, assim como o número de nevos típicos e atípicos, são também descritos como importantes fatores de risco para esta neoplasia. ${ }^{3-7}$

Apesar de representar somente $5 \%$ dos cancros cutâneos, o MMC corresponde a $75 \%$ das mortes por eles causadas. ${ }^{8}$

A sua incidência tem aumentado em Portugal, seguindo a tendência mundial. ${ }^{9-10}$ Segundo o Registo Oncológi-

'Médico interno de Medicina Geral e Familiar; Unidade de Saúde Familiar Descobertas, ACES Lisboa Ocidental-Oeiras

${ }^{2}$ Médica assistente graduada de Medicina Geral e Familiar na USF Descobertas co Nacional de 2008, a taxa de incidência padronizada à população europeia é de 6,5 casos por 100.000 habitantes, correspondendo a um aumento de $25 \%$ face a $2001 .^{9} \mathrm{Tal}$ crescimento do número de casos é não só atribuído ao aumento da exposição solar por parte da população, ${ }^{10}$ mas também a uma maior deteção desta patologia., ${ }^{411}$

A maioria dos casos de MMC primário é tratada através de excisão cirúrgica, ${ }^{12}$ atingindo-se nos países desenvolvidos uma sobrevida relativa de $83 \%$ aos cinco anos. ${ }^{13}$

Contudo, cerca de $30 \%$ destes casos pode vir a desenvolver lesões metastáticas, frequentemente a nível pulmonar. ${ }^{12}$ Apesar dos avanços, a sobrevida média dos doentes com doença metastática não ultrapassa os 12 meses. ${ }^{14}$

O risco de recidiva depende do estadio da doença, com taxas de recidiva aos dez anos a oscilar entre $10 \%$ para o estadio IA (Tla, N0, M0) e 65\% no estadio IIC (T4b, N0, M0). ${ }^{15}$

Apesar de a maioria das recidivas ter lugar nos primeiros dez anos após o diagnóstico inicial e de se considerar a sobrevida além deste período como sinónimo de cura, ${ }^{16-17}$ 
é possível encontrar na literatura um número crescente de publicações que reportam recidivas após os 10 anos, designadas como recidivas tardias. ${ }^{17-18} \mathrm{~A}$ população com este tipo de recidiva constitui um grupo de doentes que sofreu um processo de dormência tumoral, fenómeno que pode ser definido como um estadio da progressão neoplásica no qual existe doença residual, mas que, no entanto, permanece assintomática. ${ }^{19}$

Os autores apresentam o caso de um homem de 50 anos a quem foi diagnosticada doença metastática 22 anos após o diagnóstico e excisão cirúrgica de MMC. Pretende-se com o presente caso alertar para a impossibilidade de definir com segurança a cura de MMC e refletir sobre o papel do médico de família (MF) no seguimento de indivíduos com história pessoal desta neoplasia cutânea.

\section{DESCRIÇÃO DO CASO}

C.G., sexo masculino, 50 anos, natural de Lisboa, raça caucasóide, casado, ensino secundário, vendedor de profissão. Pertence a uma família nuclear na fase VI do ciclo de vida familiar de Duvall.

Apresenta, como problemas ativos, obesidade grau I, alteração da glicémia em jejum, dislipidémia mista, hipertensão arterial essencial grau 1, síndroma de apneia obstrutiva do sono e litíase renal. Como problemas passivos, destaca-se MMC da perna aos 28 anos, submetido a excisão cirúrgica completa, desconhecendo-se o seu estadiamento aquando do diagnóstico, mantendo seguimento em consulta de dermatologia até 2010 .

Encontra-se medicado habitualmente com metformina 500mg od, sinvastatina $20 \mathrm{mg}$ od, fenofibrato $145 \mathrm{mg}$ od e zofenopril 30mg od.

De hábitos, a salientar uma carga tabágica de 15 unidades maço-ano e consumo esporádico de bebidas alcoólicas.

A 17 de março de 2014 recorre à MF, em consulta programada, para mostrar controlo analítico previamente solicitado em contexto de monitorização do perfil lipídico. Trazia, contudo, outro motivo de consulta: tosse seca nos últimos dois dias, sem rinorreia, dispneia, toracalgia ou febre. Ao exame objetivo apresentava orofaringe com ligeira hiperémia sem exsudado e a auscultação pulmonar não revelava alterações. Admitiu-se infeção aguda do aparelho respiratório superior, tendo sido medicado sintomaticamente.

Um mês depois regressa em consulta aberta, por persistência da tosse, que se mantinha com as mesmas características, não evidenciando quaisquer alterações à observação. Colocou-se a hipótese de se tratar de um efeito secundário do inibidor da enzima de conversão da angio- tensina - zofenopril 30mg - que tomava para a hipertensão arterial. Pelo que se fez a sua substituição para irbesartan 150mg e se informou o utente para regressar caso não melhorasse.

Cerca de dois meses após a alteração da terapêutica, o utente retorna à consulta por manutenção da tosse seca, sem outra sintomatologia acompanhante. Após questionado, negou febre, sudorese noturna, astenia generalizada, perda ponderal (também não objetivável à observação), expectoração, hemoptises, toracalgia e dispneia. Ao exame objetivo continuava a não apresentar alterações. Nesta mesma consulta trazia consigo resultados de um controlo analítico realizado no âmbito da medicina ocupacional, que evidenciava ligeira anemia normocítica normocrómica (hemoglobina - 11,7g/dL; volume globular médio - 84,20 fL; hemoglobina corpuscular média - 27,60 pg) e um aumento da velocidade de sedimentação $(87 \mathrm{~mm} / \mathrm{h})$ sem alterações no leucograma.

Perante a clínica, a idade do utente, os seus antecedentes tabágicos, a sua profissão e as alterações analíticas foram colocadas, como hipóteses diagnósticas mais prováveis, tuberculose pulmonar e neoplasia pulmonar, pelo que foi referenciado ao serviço de urgência hospitalar para estudo imagiológico complementar e orientação diagnóstica.

No serviço de urgência realizou radiograma e, posteriormente, tomografia computorizada torácica que veio a revelar lesão nodular de características proliferativas no ápex pulmonar direito, compatíveis com neoplasia pulmonar.

O utente é então referenciado à consulta de pneumologia e, posteriormente, de cirurgia cardiotorácica, tendo sido submetido a toracotomia exploradora à direita, com resseção parcial da massa.

Os resultados histológicos revelaram metástase de MMC, 22 anos após o seu diagnóstico inicial.

Já na consulta de oncologia, onde passou a ser seguido, efetuou estudo complementar com tomografia por emissão de positrões (PET) que revelou massa pulmonar no lobo superior direito e metastização nos gânglios linfáticos mediastínicos (paratraqueais direitos e hilares homolaterais).

Em setembro de 2014 realizou três sessões de radioterapia estereotáxica corporal, tendo iniciado posteriormente ciclo de ipilimumab, que veio a terminar em dezembro de 2014. Contudo, em junho de 2015, devido a progressão da doença a nível ganglionar mediastínico, pleural direito e hepático, foi proposto para terapêutica com nivolumab em regime de administração quinzenal. Esta última foi suspensa em abril de 2016 por agravamento progressivo da função renal em contexto de nefrite tú- 
bulo-intersticial secundária ao mesmo, tendo vindo o utente a falecer em agosto de 2016.

\section{COMENTÁRIO}

A maioria das recidivas de MMC tem lugar nos primeiros dez anos após o tratamento. ${ }^{16}$ Contudo, neste caso a recidiva ocorreu 22 anos após a resseção da lesão primária, permanecendo o doente assintomático até então. O que vai ao encontro de um número cada vez maior de trabalhos publicados reportando recidivas tardias. ${ }^{17-18}$ Num conjunto de 7.104 casos de MMC, a recidiva tardia teve lugar em $2,4 \%$ dos doentes. ${ }^{18}$

No caso descrito, a localização das metástases está também de acordo com as estatísticas, que consideram o pulmão como a sede mais frequente de metastização a nível

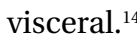

A apresentação radiológica inicial de MMC metastático é, contudo, mais frequente sob a forma de nódulos múltiplos. ${ }^{20}$ Este facto contraria o presente caso, que se apresenta como um nódulo único, o que apenas ocorre em $19 \%$ destas situações. ${ }^{20}$

O contínuo aumento do número de indivíduos que sobrevivem ao MMC e o número cada vez maior de recidivas após os dez anos, que durante muito tempo foi considerado sinónimo de cura, ${ }^{17}$ implica o estabelecimento de estratégias adequadas para o seu seguimento a longo prazo.

Os objetivos convencionais do follow-up passam pela melhoria da sobrevida através da deteção precoce da recidiva e também pela prevenção e diagnóstico atempado de uma nova neoplasia primária. ${ }^{21}$ Estas medidas pretendem-se baseadas na melhor evidência científica e respeitando critérios de custo-efetividade.

Uma melhor compreensão da dormência tumoral poderia, assim, ter importantes implicações clínicas nestes casos, permitindo determinar a frequência de recidiva tardia e respetivos fatores de risco, providenciando uma justificação para a duração do follow-up. ${ }^{17}$

Contudo, de acordo com uma revisão sistemática de $2014,{ }^{22}$ os clínicos deverão estar conscientes do quão escassa é a evidência científica que suporta muitos dos protocolos nesta área. Como consequência desta paucidade de evidência verifica-se uma considerável variabilidade nas orientações elaboradas. ${ }^{22}$

Uma revisão sistemática de 2012, que incluiu 104 estudos provenientes de oito países (Nova Zelândia, Austrália, Canadá, Alemanha, Reino Unido, Estados Unidos da América, Holanda e Suíça) e elaborados por quatro diferentes especialidades médicas (dermatologia, cirurgia, oncologia médica e medicina geral e familiar), evidenciou uma ampla variação no que diz respeito aos intervalos de vigilância propostos e às formas de avaliação recomendadas. Esta variabilidade mostrou ser maior no estadio I para o qual a frequência do follow-up oscila entre uma e seis visitas anuais, durante os primeiros dois anos. Mais consensual é a periodicidade de vigilância após cinco anos, preconizando-se a sua realização anual, exceção feita para as orientações do Reino Unido que estabelecem uma frequência adaptada às necessidades. ${ }^{23}$

No que se refere à avaliação laboratorial e imagiológica, esta é mais intensa no Reino Unido, sendo mais reduzida na Holanda. Saliente-se também que, nesta revisão, os MF, ao contrário das restantes especialidades, são os únicos que não preconizam o recurso a exames complementares. De todos os procedimentos sugeridos nos vários artigos incluídos nesta revisão sistemática, o autoexame da pele é o mais consensual, sendo recomendado por todas as especialidades..$^{23}$

Além de se tratarem das guidelines mais comummente utilizadas nas consultas de dermatologia, as recomendações da National Comprehensive Cancer Network (NCCN) são predominantemente elaboradas com recurso a evidência científica de categoria 2A. Nestas, preconiza-se para todos os doentes, independentemente do seu estadio tumoral, uma vigilância regular e para toda a vida. Estas orientações estabelecem ainda que, após o estadiamento inicial, os exames complementares deverão apenas ser solicitados de acordo com os achados clínicos obtidos no decorrer do follow-up. . $^{245}$

Ainda relativamente ao papel da medicina geral e familiar, um estudo prospetivo controlado randomizado, com 142 indivíduos, realizado na Escócia e com a duração de um ano mostrou que o follow-up de doentes de MMC conduzido pelo MF é exequível, confere maior satisfação aos doentes, permite uma maior adesão às guidelines $\mathrm{e}$ não apresentou efeitos adversos ao nível do estado de saúde, ansiedade ou depressão, quando comparado com o follow-up tradicional a nível hospitalar. ${ }^{26}$

Não obstante, é possível encontrar na literatura trabalhos que visam determinar a acuidade de diagnóstico de neoplasias cutâneas por parte dos MF, particularmente quando comparados com dermatologistas.

Uma revisão sistemática, que incluiu 32 estudos (prospetivos e retrospetivos) realizados entre 1966 e 1999, concluiu que a informação disponível é inadequada para demonstrar diferenças entre dermatologistas e MF no diagnóstico de lesões sugestivas de MMC. ${ }^{27}$

Já um estudo prospetivo realizado em França no ano de 
2000 (como tal, não incluído na revisão sistemática supra referida), e que avaliou 590 casos clínicos, concluiu que os dermatologistas não só apresentam maior acuidade no diagnóstico de MMC, como também orientam estes casos de modo mais célere e apropriado, quando comparados com outros médicos, resultando, assim, num nível de Breslow mais reduzido e, portanto, num melhor prognóstico. ${ }^{28}$

Ao encontro destes resultados está um estudo observacional transversal, realizado em Itália e publicado em 2002 que, através da aplicação de um questionário de escolha múltipla com casos clínicos e imagens, avaliou a capacidade de 327 MF ao nível do diagnóstico precoce de neoplasias cutâneas, respetivo diagnóstico diferencial e tratamento e que veio a concluir que uma parte significativa dos MF não se sente confiante na sua capacidade de reconhecimento de neoplasias cutâneas, tendo identificado também défices de conhecimento nesta área. ${ }^{29}$

À data, os autores desconhecem estudos semelhantes realizados em Portugal, pelo que estes dados devem ser encarados à luz da realidade dos respetivos países.

O presente caso é também uma fiel ilustração das várias competências inerentes à medicina geral e familiar. ${ }^{30}$

Evidencia o recurso a aptidões específicas para a resolução de problemas: o episódio de cuidados inicia-se com quadro indiferenciado e precoce, composto por apenas um sintoma, cuja marcha diagnóstica considerou a incidência e prevalência das patologias. Perante uma apresentação aguda de tosse foram consideradas as causas mais frequentes neste contexto, como a infeção do aparelho respiratório superior. ${ }^{31}$

Espelha uma abordagem abrangente, uma vez que este caso se inicia numa consulta em que, paralelamente ao seguimento de um problema crónico (a dislipidémia), se abre um novo episódio de cuidados referente a um sintoma agudo com apenas dois dias de evolução, mas que acaba por alcançar a cronicidade.

Reflete ainda o papel do MF enquanto gestor de cuidados: ao não compartimentar a sua atuação, tendo observado o resultado das análises solicitadas por outro prestador, abriram-se novas hipóteses diagnósticas e modificouse a conduta, mobilizando-se várias valências a nível dos cuidados de saúde secundários e terciários, orientando-se e disponibilizando-se ao paciente os serviços adequados.

Salienta também a importância dos cuidados longitudinais e de uma boa comunicação entre médico e doente, mostrando a importância de "estender uma rede de segurança”, assim designada por Mendes Nunes: consciencializando o doente para uma evolução clínica menos pro- vável, mas ainda assim possível, protegeu-se não só o doente (que sentiu abertura para regressar à consulta por mais de uma vez, expressando a persistência da sua queixa) como também o próprio clínico, reduzindo as probabilidades de ser acusado de má prática. ${ }^{32}$

Em conclusão, o presente caso visa sensibilizar para a ocorrência da recidiva tardia de MMC, contrariando o conceito de cura após dez anos livres de doença, enfatizando a necessidade do seguimento destes doentes para toda a vida. Assim, e considerando a continuidade de cuidados prestada pelo MF, torna-se premente que este, a par do dermatologista, desempenhe um papel crucial na monitorização de tais casos, estando ciente que novos sintomas ou achados físicos poderão relacionar-se com o diagnóstico prévio de MMC.

\section{REFERÊNCIAS BIBLIOGRÁFICAS}

1. Russo A, Ficili B, Candido S, Pezzino FM, Guarneri C, Biondi A, et al. Emerging targeted therapies for melanoma treatment (review). Int J Oncol. 2014;45(2):516-24

2. Chang YM, Barrett JH, Bishop DT, Armstrong BK, Bataille V, Bergman W, et al. Sun exposure and melanoma risk at different latitudes: a pooled analysis of 5700 cases and 7216 controls. Int J Epidemiol. 2009;38(3):814-30.

3. Gandini S, Sera F, Cattaruzza MS, Pasquini P, Abeni D, Boyle P, et al. Meta-analysis of risk factors for cutaneous melanoma: I. Common and atypical naevi. Eur J Cancer. 2005;41(1):28-44.

4. Gandini S, Sera F, Cattaruzza MS, Pasquini P, Picconi O, Boyle P, et al. Metaanalysis of risk factors for cutaneous melanoma: II. Sun exposure. Eur J Cancer. 2005;41(1):45-60.

5. Gandini S, Sera F, Cattaruzza MS, Pasquini P, Zanetti R, Masini C, et al. Metaanalysis of risk factors for cutaneous melanoma: III. Family history, actinic damage and phenotypic factors. Eur J Cancer. 2005;41(14):2040-59.

6. Gandini S, Autier P, Boniol M. Reviews on sun exposure and artificial light and melanoma. Prog Biophys Mol Biol. 2011;107(3):362-6.

7. Whiteman DC, Whiteman CA, Green AC. Childhood sun exposure as a risk factor for melanoma: a systematic review of epidemiologic studies. Cancer Causes Control. 2001;12(1):69-82

8. Siegel R, Ma J, Zou Z, Jemal A. Cancer statistics, 2014. CA Cancer J Clin. 2014;64(1):9-29.

9. ROR-Centro. Registo oncológico nacional, 2008 [Internet]. Coimbra: Instituto Português de Oncologia de Coimbra Dr. Francisco Gentil, EPE; 2014. Available from:http://www.rorcentro.com.pt/Data/RORCentro/Publica\%C3\%A7\%C3\% A3o_Nacional_2008.pdf

10. Erdmann F, Lortet-Tieulent J, Schüz J, Zeeb H, Greinert R, Breitbart EW, et al. International trends in the incidence of malignant melanoma 1953-2008: are recent generations at higher or lower risk? Int J Cancer. 2013;132(2):385-400.

11. Bataille V, de Vries E. Melanoma, Part 1: epidemiology, risk factors, and prevention. BMJ. 2008;337:a2249.

12. Essner R, Lee JH,Wanek LA, Itakura H, Morton DL. Contemporary surgical treatment of advanced-stage melanoma. Arch Surg. 2004;139(9):961-6.

13. Crocetti E, Mallone S, Robsahm TE, Gavin A, Agius D, Ardanaz E, et al. Survival of patients with skin melanoma in Europe increases further: results of the EUROCARE-5 study. Eur J Cancer. 2015;51(15):2179-90.

14. Tas F. Metastatic behavior in melanoma: timing, pattern, survival, and influen- 
cing factors. J Oncol. 2012;2012:ID647684.

15. Turner RM, Bell KJ, Morton RL, Hayen A, Francken AB, Howard K, et al. Optimizing the frequency of follow-up visits for patients treated for localized primary cutaneous melanoma. J Clin Oncol. 2011;29(35):4641-6.

16. Tsao $\mathrm{H}$, Cosimi AB, Sober AJ. Ultra-late recurrence (15 years or longer) of cutaneous melanoma. Cancer. 1997;79(12):2361-70.

17. Faries MB, Steen S, Ye X, Sim M, Morton DL. Late recurrence in melanoma: clinical implications of lost dormancy. J Am Coll Surg. 2013;217(1):27-34.

18. Crowley NJ, Seigler HF. Late recurrence of malignant melanoma: analysis of 168 patients. Ann Surg. 1990;212(2):173-7.

19. Aguirre-Ghiso JA. Models, mechanisms and clinical evidence for cancer dormancy. Nat Rev Cancer. 2007;7(11).834-46.

20. Smyth EC, Hsu M, Panageas KS, Chapman PB. Histology and outcomes of newly detected lung lesions in melanoma patients. Ann Oncol. 2012;23(3):577-82.

21. Carter D, Afzali HH, Street J, Bessen T, Neuhaus S. Melanoma follow up: time to generate the evidence. Aust Health Rev. 2013;37(4):501-3.

22. Marciano NJ, Merlin TL, Bessen T, Street JM. To what extent are current guidelines for cutaneous melanoma follow up based on scientific evidence? Int J Clin Pract. 2014;68(6):761-70.

23. Cromwell KD, Ross MI, Xing Y, Gershenwald JE, Royal RE, Lucci A. Variability in melanoma post-treatment surveillance practices by country and physician specialty: a systematic review. Melanoma Res. 2012;22(5):376-85.

24. Carlson RW, Larsen JK, McClure J, Fitzgerald CL, Venook AP, Benson AB 3rd, et al. International adaptations of NCCN clinical practice guidelines in oncology. J Natl Compr Canc Netw. 2014;12(5):643-8.

25. Coit DG, Thompson JA, Algazi A, Andtbacka R, Bichakjian CK, Carson WE 3rd, et al. NCCN guidelines insights: melanoma, version 3.2016. J Natl Compr Canc Netw. 2016;14(8):945-58.
26. Murchie P, Nicolson MC, Hannaford PC, Raja EA, Lee AJ, Campbell NC. Patient satisfaction with GP-led melanoma follow-up: a randomised controlled trial. Br J Cancer. 2010;102(10):1447-55.

27. Chen SC, Bravata DM, Weil E, Olkin I.A comparison of dermatologists' and primary care physicians' accuracy in diagnosing melanoma. Arch Dermatol. 2001;137(12):1627-34.

28. Richard MA, Grob JJ, Avril MF, Delaunay M, Gouvernet J, Wolkenstein P, et al. Delays in diagnosis and melanoma prognosis (II): the role of doctors. Int J Cancer. 2000;89(3):280-5.

29. Offidani A, Simonetti O, Bernardini ML, Alpagut A, Cellini A, Bossi G. General practitioners' accuracy in diagnosing skin cancers. Dermatology. 2002;205 (2):127-30.

30. European Academy of Teachers in General Practice/Family Medicine (EURACT). A definição europeia de medicina geral e familiar: versão reduzida. Rev Port Clin Geral. 2005;21(5):511-6.

31. Pratter MR. Cough and the common cold:ACCP evidence-based clinical practice guidelines. Chest. 2006;129(1 Suppl):72S-4S.

32. Nunes JM. Comunicação em contexto clínico. Lisboa: Bayer Health Care; 2007.

\section{CONFLITO DE INTERESSES}

Os autores declaram não ter conflitos de interesses.

\section{ENDEREÇO PARA CORRESPONDÊNCIA}

Diogo Tomás Gamito Barata de Almeida

E-mail: diogo_dealmeida@hotmail.com

Recebido em 24-02-2016

Aceite para publicação em 27-10-2016

\section{ABSTRACT}

\section{LATE RECURRENCE OF CUTANEOUS MELANOMA: CASE REPORT}

Introduction: Ten-year survival for patients with cutaneous malignant melanoma (CMM) is considered to be synonymous with cure. However, there are reports of late recurrence after this period. This report stresses the importance of awareness of late recurrences and reflects on the role of the family physician (FP) in timely diagnosis.

Case description: A 50-year-old man, who was a smoker with history of arterial hypertension and the excision of a CMM at 28 years of age, came to see his FP complaining of a dry cough for two days. An upper respiratory tract infection was diagnosed and treated symptomatically. One month later he returned due to the persistence of the cough. There were no other abnormal signs or symptoms. The cough was presumed to be a side effect of Zofenopril prescribed for control of arterial hypertension. This was stopped and replaced with Irbesartan. He returned two months later with the same complaint. There were no complaints of fever, sweating, chest pain, haemoptysis, or weight loss. Physical examination was normal. The erythrocyte sedimentation rate, ordered by his occupational health doctor, was $87 \mathrm{~mm} / \mathrm{h}$. The diagnoses of pulmonary tuberculosis or lung cancer were considered. He was referred to the local emergency room, where a thoracic computerized tomography scan was performed. It revealed a proliferative nodular lesion on the left lung apex, compatible with lung cancer. The patient was referred for a pulmonary consultation, and was then admitted to hospital for partial resection of the mass. The histological results showed a metastasis of CMM, 22 years after the initial diagnosis.

Comment: There are a growing number of reports of late recurrences of CMM. Early detection should be a concern for physicians. The FP can play an important role on monitoring these cases given the longitudinal continuity of care they provide.

Keywords: Melanoma; Late Recurrence. 